\title{
Evaluation of olive mill waste as substrate for carotenoid production by Rhodotorula mucilaginosa
}

\author{
Carolina Ghilardi ${ }^{1}$, Paola Sanmartin Negrete ${ }^{3}$, Amalia Antonia Carelli ${ }^{1,2}$ and Virginia Borroni $i^{1,3^{*}}$ (D)
}

\begin{abstract}
The "alperujo" is a waste from the olive oil industry with great potential for valorization. It has a high organic load, with the presence of valuable compounds such as biophenols and sugars. The use of this waste can be thought of as a biorefinery from which different compounds of high added value can be obtained, whether they are present in the "alperujo" such as biophenols or can be generated from the "alperujo". Therefore, the production of carotenoids by Rhodotorula mucilaginosa was evaluated using the liquid fraction of 'alperujo' (Alperujo Water, AW) or an aqueous extract (AE) of "alperujo" at different concentrations (5, 10, 20 and 30\% w/N) as substrates. The AEs had an acidic pH, a total sugar concentration ranging from 1.6 to $7.6 \mathrm{~g} / \mathrm{L}$, a polyphenols content from 0.4 to $2.9 \mathrm{~g} / \mathrm{L}$ and a significant amount of proteins (0.5-3 g/L). AW is similar in composition as 30\% AE, but with a higher amount of total sugars. Rh. mucilaginosa was able to grow at the different mediums with consumption of glucose and fructose, a reduction in protein content and alkalinization of the medium. Maximum total carotenoid production $(7.3 \pm 0.6 \mathrm{mg} / \mathrm{L}) \mathrm{was}$ achieved at AW, while the specific production was higher when the yeast grew at AW or at $30 \% \mathrm{AE}(0.78 \pm 0.06$ and $0.73 \pm 0.10 \mathrm{mg} / \mathrm{g}$ of biomass, respectively). Torulene and torularhodin were the main carotenoids produced. Polyphenol content did not change; thus, it is still possible to recover these compounds after producing carotenoids. These results demonstrate the feasibility of using alperujo-based mediums as cheap substrates to produce torularhodin and torulene and to include this bioprocess as a step in an integral approach for alperujo valorization.
\end{abstract}

Keywords: Alperujo, Carotenoids, Torulene, Torularhodin, Waste valorization, Microbial growth

\section{Introduction}

Nowadays, olive oil is obtained by two- and threephase centrifugation systems which in most countries replace the traditional pressing. These systems save water and energy and improve oil recovery; however, different by-products are generated that need to be appropriately managed (Morillo et al. 2009). With the two-phase centrifugation system, only one semi-solid residue called two-phase olive mill waste (TPOMW)

\footnotetext{
*Correspondence: mvirborroni@gmail.com

${ }^{3}$ Instituto de Tecnología en Polímeros y Nanotecnología

(ITPN-UBA-CONICET), Facultad de Arquitectura, Diseño y Urbanismo

(FADU), Universidad de Buenos Aires (UBA), Ciudad Universitaria,

1428 Buenos Aires, Argentina

Full list of author information is available at the end of the article
}

or "alperujo" is produced. It is estimated that $850 \mathrm{~kg}$ of "alperujo" are produced per one ton of olives processed (Serrano et al. 2017). It has $\sim 65 \%$ moisture content, a slightly acidic $\mathrm{pH}$ and a high content of organic matter composed of lignin, cellulose, hemicellulose, fats, polyphenols and carbohydrates. This high organic load, if applied in high quantities and during long periods, contaminates soil and water (Killi et al. 2014; Monetta et al. 2012). However, since alperujo is essentially olive pulp, olive stone and vegetation water, it contains many of the beneficial components of the olive fruit and with the appropriate treatment, it could be considered as a by-product of the olive industry (Serrano et al. 2017; Dermeche et al. 2013). Recent approaches to valorize alperujo point to the recovery 
of some of these valuable compounds like hydroxytyrosol and tyrosol, both recognized potent antioxidants (Rubio-Senent et al. 2013; Fernández-Prior et al. 2020). However, even with these treatments, alperujo may be under-exploited as most lignocellulosic feedstocks (Unrean 2016). An interesting alternative for alperujo valorization is to use it as a substrate for the growth of microorganisms of biotechnological interest. This strategy provides the possibility of producing high value-added products such as biopolymers, enzymes and pigments with a concomitant reduction of the organic load (Borroni et al. 2017; Moya Ramírez et al. 2016; Elisashvili et al. 2018; Zerva et al. 2017).

Carotenoids represent a valuable class of lipid molecules exploited at an industrial scale as additives in pharmaceutical, chemical, food and feed products. Their industrial market is expected to reach about $\$ 2.0$ billion by 2022 (McWilliams 2018). At the biological level, carotenoids are antioxidant pigments that prevent oxidative damage to lipid membranes as well as some of them are precursors of vitamin A (Rodriguez-Concepcion et al. 2018). They are synthesized by plants, algae, yeast, fungi and bacteria, but not by animals, which must obtain them from the diet (Rodriguez-Concepcion et al. 2018). Among the yeasts, the genus Rhodotorula is well known to produce a variety of carotenoids including $\beta$-carotene, torulene and torularhodin (Tang et al. 2019). Although $\beta$-carotene is one of the most studied carotenoids, in the last years, torularhodin and torulene have gain attention because they are stronger antioxidants and have antimicrobial and antitumoral activities (Zoz et al. 2015; Yoo et al. 2016).

Several studies have demonstrated the feasibility of producing carotenoids from low-cost substrates (Kaur et al. 2019; Sharma and Ghoshal 2020; Urnau et al. 2019). In this work, we explore the production of carotenoids, in particular, torulene and torularhodin, by a strain of Rhodotorula mucilaginosa using different medium derived from alperujo as cheap substrates.

\section{Materials and methods Microorganism}

Rhodotorula mucilaginosa was kindly provided by the Instituto de Botánica "Carlos Spegazzini" and it was maintained in agar slants at $4{ }^{\circ} \mathrm{C}$ until required. The identification of the yeast strain was performed by the Departamento de Micología, Instituto Nacional de Enfermedades Infecciosas Dr. Carlos G. Malbrán by phenotypic tests, MALDI-TOF MS (Matrix-Assisted Laser Desorption/Ionization-Time of Flight) and DNA sequencing of the D1/D2 domain of $26 \mathrm{~s}$ ribosomal DNA.

\section{Two-phase olive mill waste (TPOMW) samples}

Arbequina fresh TPOMW samples were provided by an oil processing plant located in Coronel Dorrego, province of Buenos Aires (Argentina). The samples were stored at $-20{ }^{\circ} \mathrm{C}$ until further use.

\section{Culture medium preparation}

Alperujo Water (AW) was obtained by centrifuging fresh alperujo at $1372 g$ for $20 \mathrm{~min}$. The liquid phase was separated, filtered and sterilized by autoclaving at $120{ }^{\circ} \mathrm{C}$ for $15 \mathrm{~min}$. Aqueous extracts (AE) were prepared from freeze-dried and defatted alperujo in concentrations of 5 , 10, 20 and 30\% (w/V) according to (Borroni et al. 2017). In brief, the corresponding amount of TPOMW was extracted with continuous agitation for $2 \mathrm{~h}$ at $4{ }^{\circ} \mathrm{C}$ with distilled water. The obtained aqueous extract (AE) was then centrifuged, filtered and sterilized by autoclaving at $120^{\circ} \mathrm{C}$ for $15 \mathrm{~min}$.

\section{Analytical procedures}

Total phenolic content was determined spectrophotometrically at $725 \mathrm{~nm}$ according to the Folin-Ciocalteu method using caffeic acid as standard (Gutfinger 1981). Glucose was measured with an enzymatic-Glicemia kit, kindly provided by Wiener Lab (Rosario, Argentina). The pH was measured with an Altronix TPX-I electrode. Protein concentration was determined by the Lowry method (Lowry et al. 1951) previous precipitation with trichloroacetic acid. Dry cell weight was determined at $85^{\circ} \mathrm{C}$ using a Kern DLB 160-3A thermobalance (Kern, Germany). The number of yeasts was counted in a Neubauer chamber using an inverted microscope Zeiss Primo Star (Carl Zeiss, Germany). A non-inoculated flask (W/In) was included as a control in all experiments and treated as the inoculated samples.

Determination of sugar composition of the different mediums was performed by a Waters Alliance e2695 HPLC (Waters Corp., Milford, MA, USA) on an ion exclusion Rezex ${ }^{\mathrm{TM}} \mathrm{RCM}$-Monosaccharide $\mathrm{Ca}^{+2}$ analytical column (Phenomenex, Torrance, CA, USA) kept at $60{ }^{\circ} \mathrm{C}$, using an injection volume of $5 \mu \mathrm{L}$ and a flow rate of $0.6 \mathrm{~mL} / \mathrm{min}$. The mobile phase consisted of HPLC-grade deionized water. Peaks were monitored by changes in the refractive index with a Waters 2414 Refractive Index (RI) detector (Waters Corp., Milford, MA, USA). Calibration curves constructed with pure standards (Anedra, Buenos Aires, Argentina) were used to determine the concentration of glucose $\left(r^{2}=0.992\right)$, fructose $\left(r^{2}=0.996\right)$, mannitol $\left(r^{2}=0.997\right)$, stachyose $\left(r^{2}=0.993\right)$ and sucrose $\left(r^{2}=0.983\right)$. The detection limit was calculated from the calibration curves according to (Hadjikinova et al. 2017) 
and was $0.035 \mathrm{mg} / \mathrm{mL}$ for glucose, $0.020 \mathrm{mg} / \mathrm{mL}$ for fructose, $0.025 \mathrm{mg} / \mathrm{mL}$ for mannitol, $0.015 \mathrm{mg} / \mathrm{mL}$ for sucrose and $0.013 \mathrm{mg} / \mathrm{mL}$ for stachyose.

\section{Production, extraction, and quantification of carotenoids}

Cultures were performed in 250-mL Erlenmeyer flasks containing $50 \mathrm{~mL}$ of the desired concentration of $\mathrm{AE}$ and incubated for 6 days at $30{ }^{\circ} \mathrm{C}$ under orbital shaking (150 rpm). Samples were taken on days 0, 1, 2, 3, 4 and 6. To measure glucose, total phenolics, $\mathrm{pH}$ and biomass, $2 \mathrm{~mL}$ of culture was removed under sterile conditions. The medium was centrifugated at $1372 g$ for $15 \mathrm{~min}$, the biomass separated and the supernatant was filtrated and stored at $-20^{\circ} \mathrm{C}$ until required.

For carotenoid extraction, the cells were incubated with dimethyl sulfoxide at $50{ }^{\circ} \mathrm{C}$ for $30 \mathrm{~min}$ and thereafter extracted with $3 \mathrm{~mL}$ acetone until the residual cell pellets were colorless. The supernatant was separated by centrifugation at $1372 \mathrm{~g}$ during $15 \mathrm{~min}$ and acetone extracts were combined. Pooled extracts were collected in amber flasks, the solvent was evaporated under a nitrogen stream and carotenoids were suspended in 3-mL acetone (Colet et al. 2015). Quantification was performed by measuring absorbance at $450 \mathrm{~nm}$ in a Shimadzu UV-160A spectrophotometer. A calibration curve was prepared with $\beta$-carotene in acetone and the result was expressed as $\beta$-carotene equivalents. Each culture condition was run in triplicate.

Separation of carotenoids was achieved by a Waters Alliance e2695 HPLC on a C-18 reverse-phase analytical column (Waters Spherisorb ODS2 $5 \mu \mathrm{m}$, $4.6 \mathrm{~mm} \times 250 \mathrm{~mm}$ ) kept at $25{ }^{\circ} \mathrm{C}$, using an injection volume of $10 \mu \mathrm{L}$ and a flow rate of $1 \mathrm{~mL} / \mathrm{min}$. The mobile phase consisted of a binary gradient of acetone and water. The initial composition was $75 \%$ acetone, which was linearly increased to $95 \%$ acetone in $10 \mathrm{~min}$. It was maintained at this composition for the next $7 \mathrm{~min}$, then raised to $100 \%$ acetone in $3 \mathrm{~min}$ and held for $10 \mathrm{~min}$. The initial composition was reached in $5 \mathrm{~min}$. (Fernandez-Orozco et al. 2013). Peaks were monitored at $450 \mathrm{~nm}$ and online spectra were recorded between 320 and $700 \mathrm{~nm}$ with a Waters 2998 Photodiode Array Detector. A calibration curve was prepared with a $\beta$-carotene standard from Sigma Chemical Co. (St. Louis, MO) and the concentration of each carotenoid was expressed as $\beta$-carotene equivalents. Tentative identification was performed by comparing the retention time and UV-Vis spectra with literature data (Weber et al. 2007; Britton and Young 1993). The \%III/II was calculated as the relationship between the peak heights of the longest-wavelength absorption band (III) and the middle absorption band (II), taking as a baseline the minimum between the two peaks (Rodriguez-Amaya and Kimura 2004).

\section{Seed germination test}

Seed germination was carried out in glass Petri dishes (100-mm diameter) with filter paper (140-mm Whatman No. 1 filter) on the bottom. Twenty seeds of Lactuca sativa were added in dishes contained $5 \mathrm{~mL}$ of distilled water (control) or the corresponding medium (AW or AE at different concentrations) before and after Rh. mucilaginosa growth. Petri dishes were maintained for $72 \mathrm{~h}$ under dark conditions at $20{ }^{\circ} \mathrm{C}$ and the number of germinated seeds was counted every day. Germination was determined by observation under a magnifying glass, considering that a seed is germinated when the root is longer than $1 \mathrm{~mm}$ (Di Salvatore et al. 2008).

\section{Sugar and nitrogen assimilation test}

Carbon assimilation test was performed using a minimal nitrogen medium supplemented with stachyose, raffinose, sucrose, glucose, fructose or mannitol as sole carbon source at a concentration of $20 \mathrm{~g} / \mathrm{L}$ (Wickerham and Burton 1948). Nitrogen assimilation test was performed using a minimal medium with glucose supplemented with $\mathrm{NaNO}_{3},\left(\mathrm{NH}_{4}\right)_{2} \mathrm{SO}_{4}$, urea or bovine serum albumin (BSA) as sole nitrogen source at a concentration of $1 \mathrm{~g} / \mathrm{L}$ (Wickerham 1946). Growth was determined spectrophotometrically by measuring O.D. at $600 \mathrm{~nm}$ against a medium inoculated with yeasts without the addition of any carbon or nitrogen source.

\section{Statistical analysis}

Analysis of variance (ANOVA) followed by post hoc Tukey's multiple comparisons test was performed using GraphPad Prism version 6.00 for Windows (GraphPad Software, La Jolla, California, USA, www.graphpad.com). A threshold of $p=0.05$ was set as a significance level to compare means.

\section{Results and discussion Characterization of alperujo water and aqueous extract of alperujo}

Figure 1 shows the main physicochemical characteristics of different concentrations of AE and from AW used as a growth medium for yeast development. Although the composition of olive wastes varies depending on the olive variety, climate conditions, cultivation practices and storage time, we found values were consistent with other reports (Giannoutsou et al. 2004; Dermeche et al. 2013; Serrano et al. 2017). All mediums are acidic $(\mathrm{pH}=5.15-5.39)$ and have a high polyphenol content (Fig. 1a, b). Recent reports optimize pH values for carotenoid production by $R h$. mucilaginosa in agro-industrial wastes to be 6.1, higher than the $\mathrm{pH}$ of our mediums (Sharma and Ghoshal 2020). These factors may preclude 


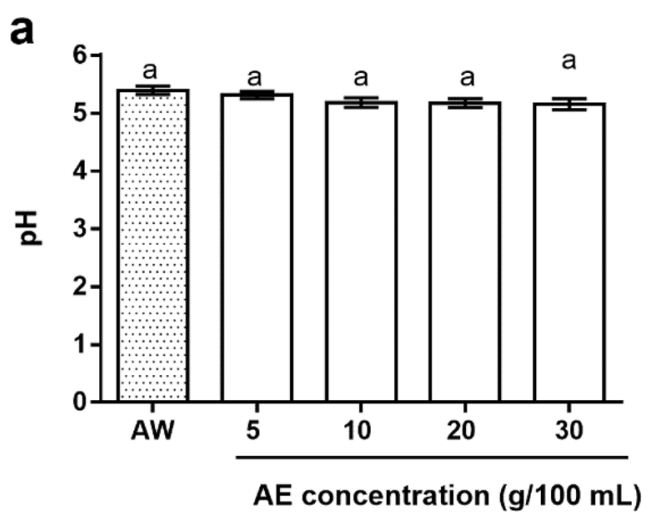

C

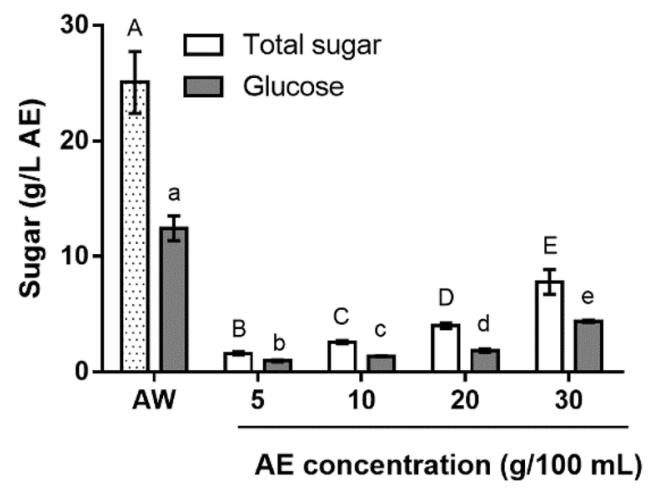

b

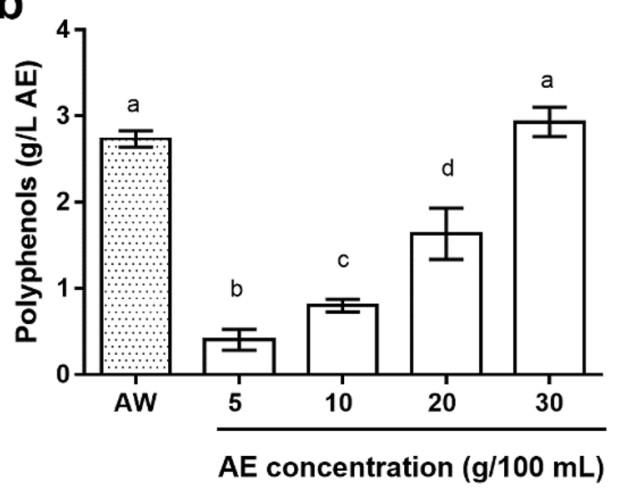

d

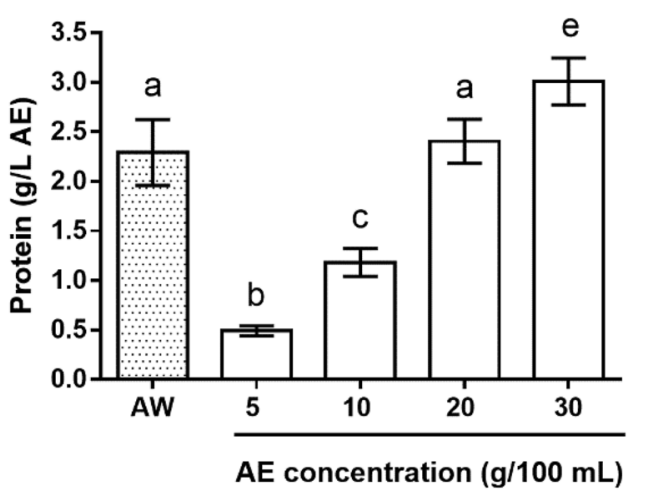

Fig. 1 Physicochemical characteristics of different mediums. pH (a), polyphenol content (b), Total sugar and glucose contents (c) and protein contents (d) were measured in the different mediums used as substrate for carotenoid production by Rh. mucilaginosa. Bars show the mean and S.D. values $(n=4)$; different letters indicate statistically significant differences $\left(p^{<} 0.01\right)$

yeast growth; however, an important amount of total sugars-including glucose-and a significant amount of protein are present in the extract (Fig. 1c, d). There is a variety of sugars solubilized in the mediums (Table 1), among them glucose and mannitol are the most abundant. It would be advantageous if Rh. mucilaginosa could use these compounds as carbon and nitrogen sources to grow and produce carotenoids efficiently. Indeed, metabolic tests showed that $R h$. mucilaginosa can use proteins as nitrogen source, although less efficiently than peptone, urea or $-\mathrm{NO}_{3}{ }^{-}$(Additional file 1: Fig. S1a). Also, it is able to grow using all the sugars present in alperujo as the sole C source (Additional file 1: Fig. S1b). Therefore, Rh. mucilaginosa has the ability to assimilate the nutrients present in the alperujo-based mediums, a desirable characteristic of the strain.

Since alperujo has a $65 \%$ water content, the liquid fraction of alperujo (AW) is expected to be similar in composition as $30 \% \mathrm{AE}$. Interestingly, AW has much more total sugar and glucose than the AE (Fig. 1c). This excess in carbon compounds could be an advantage for producing carotenoids since they are produced when there is a high $\mathrm{C} / \mathrm{N}$ ratio (Elfeky et al. 2019). It is important to note that AW is prepared by simply centrifuging fresh alperujo and separating the liquid fraction. Therefore, it can be obtained in line with olive oil production without the addition of water. However, AEs are prepared by reconstituting the appropriate amount of freeze-dried alperujo with distilled water. Since olive oil production is a stational process, alperujo is produced intensively during a short period. The use of dried alperujo has the advantage to facilitate its storage and transport.

\section{Characterization of Rh. mucilaginosa growth at different medium}

Rhodotorula mucilaginosa is able to grow in AW and at all AE concentrations studied (Fig. 2). With these sampling times, the lag period was evident only at AW and $30 \% \mathrm{AE}$, probably due to the high levels of toxic compounds present in the mediums (Fig. 2 a). The time to reach the stationary phase augments as the $\mathrm{AE}$ concentration increases, being 1 day at 5\% and $10 \% \mathrm{AE}$ and 2 days at 20\%, 30\% AE and AW (Fig. 2a). For all $\mathrm{AE}$ concentration tested, at 5 days, deleterious effects 


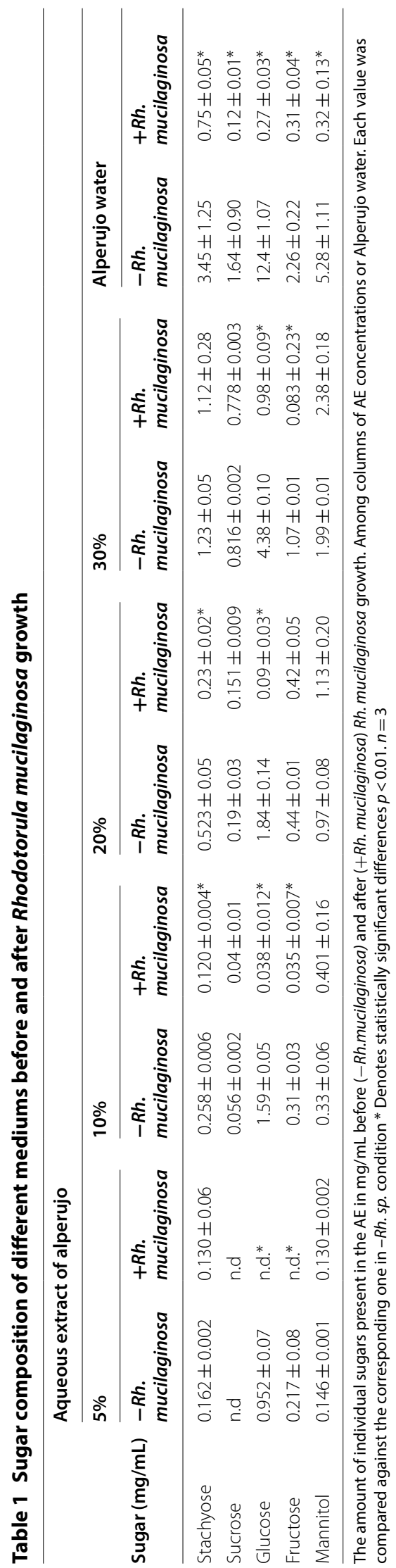




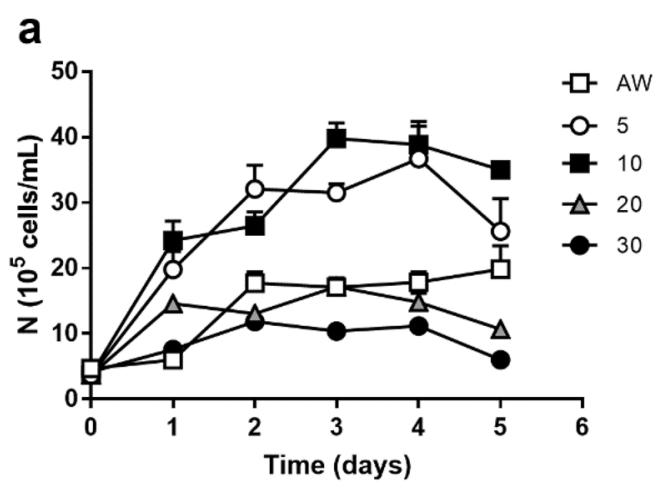

C

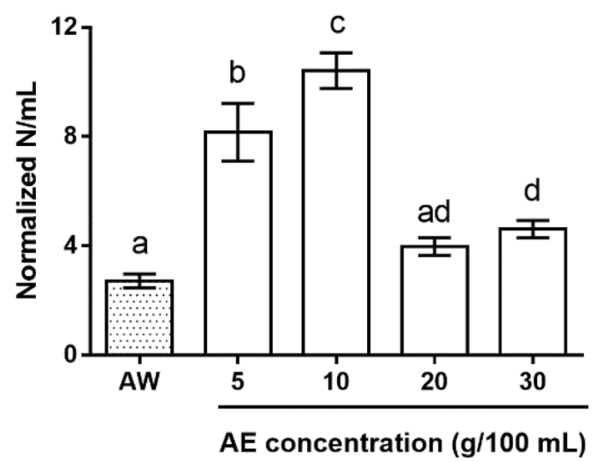

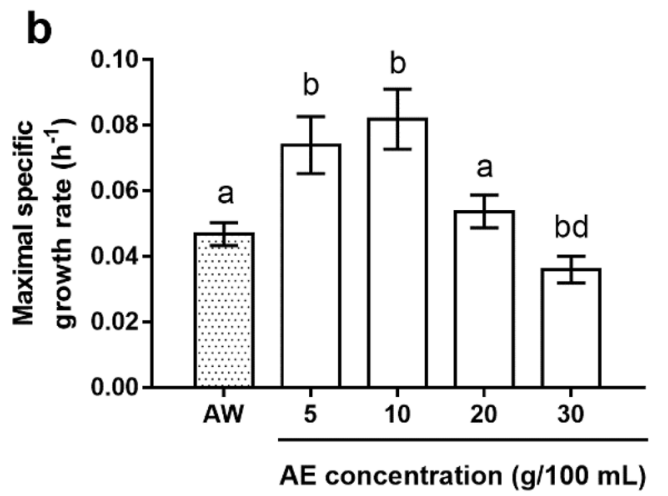

d

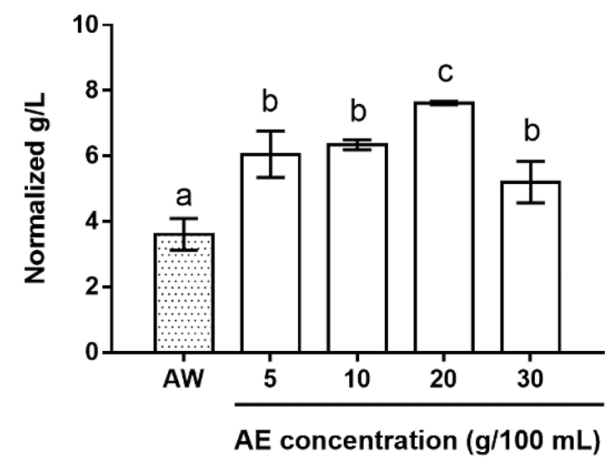

Fig. 2 Characteristics of Rh. mucilaginosa growth at different mediums. Time course of Rh. mucilaginosa growth (a) and maximal specific growth rate (b) at different AE concentration. Normalized (final/initial) biomass obtained at 4 days of culture, measured as number of cells (c) or dry weight (d). Data are shown as the mean and S.D. $(n=4)$; different letters indicate statistically significant differences $\left(p^{<} 0.01\right)$

on $R$ h. mucilaginosa viability are observed, and thus, next experiments were performed for 4 days. The maximal specific growth rate was markedly higher at $5 \%$ and $10 \% \mathrm{AE}$; whereas at $20 \%, 30 \%$ and AW Rh. mucilaginosa slows down its growth (Fig. 2b). The number of yeasts produced also has a maximum at $10 \% \mathrm{AE}$ and decreases at lower and higher $\mathrm{AE}$ concentrations and at AW (Fig. 2c). This behavior of the biomass produced was observed before with a strain of Rh. mucilaginosa grown on an algae extract and with other microorganisms (Leyton et al. 2019; Morillo et al. 2006). At 5\% AE, the amount of available nutrients may limit the growth; while at more concentrated mediums (20 and 30\% AE and $\mathrm{AW}$ ), the excess of toxic compounds, i.e., polyphenols, may inhibit yeast growth. Interestingly, the biomass measured as the dry weight has a maximum at $20 \%$ AE; whereas, the number of yeasts shows the highest value at $10 \% \mathrm{AE}$ (Fig. 2d). This implies that at $10 \%$ the nutrient balance favors cell division; while at higher concentration, accumulation of reserve substance may be responsible for the increase in weight rather than the number of cells (Schneider et al. 2013; Elfeky et al. 2019).

\section{Characterization of alperujo water and aqueous extract of alperujo after $R$ h. mucilaginosa growth}

$R h$. mucilaginosa growth increases the $\mathrm{pH}$ of the medium and this effect is more marked at low AE concentrations (Fig. 3a). The assimilation of organic acids in addition to the deamination of amino acids will result in alkalinisation of the medium. A similar result was observed with Rh. glutinis grown on potato wastes and with a strain of Rh. mucilaginosa used to treat olive mill wastewater (Jarboui et al. 2012) (Kot et al. 2017). Protein content decreases at all AE concentrations and in AW indicating that $R h$. mucilaginosa is able to use these compounds as a nitrogen source (Fig. 3b, Additional file 1: Fig S1a). Protein consumption is larger at $10 \% \mathrm{AE}(\sim 65 \%)$, probably related to the fact that at this concentration the maximal growth rate is observed and a larger number of cells are produced (Fig. 2b). Interestingly, not all the protein present could be consumed and larger quantities remain in the medium, a situation that is more evident at 20 and $30 \% \mathrm{AE}$, where only $25 \%$ is used. In the case of $\mathrm{AW}, \sim 50 \%$ of the soluble proteins remain in the medium after Rh. mucilaginosa growth. Thus, although a nitrogen source is present in the medium, the yeast experiments 

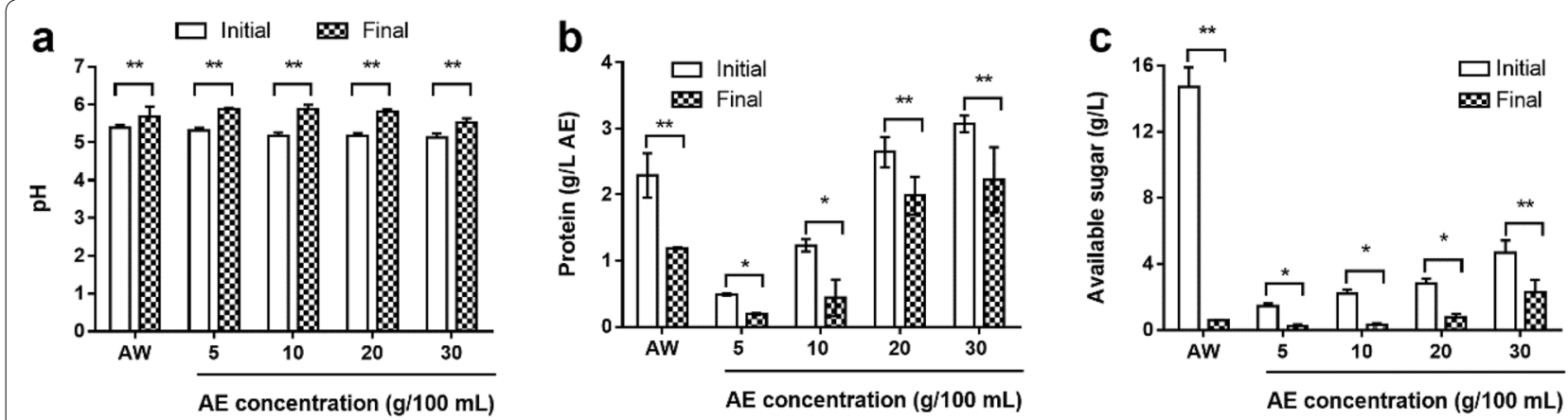

Fig. 3 Changes in physicochemical parameters of different mediums after Rh. mucilaginosa growth. $\mathrm{pH}$ (a), protein (b) and available sugars (c) were measured in the different AE concentrations before (Initial, empty bars) and after 4 days in culture (Final, dotted bars) Rh. mucilaginosa growth. Bars show the mean and S.D. $(n=4),{ }^{*} p^{<} 0.01,{ }^{* *} p<0.001$

a nitrogen deficiency, a condition known to favor carotenoid synthesis. Similar results were found with $R h$. glutinis grown with different nitrogen sources, where cellular carotenoid accumulation was enhanced significantly in a high $\mathrm{C} / \mathrm{N}$ ratio compared to a low $\mathrm{C} / \mathrm{N}$ ratio (Elfeky et al. 2019). This inability of Rhodotorula to use efficiently the protein presents as nitrogen source may limit yeast growth and shift the metabolism to accumulate carbon excess as reserve substances. Available sugars were also consumed at different extents according to the medium (Fig. 3c). Rhodotorula mucilaginosa consumed between 80 and $85 \%$ of sugars when growing at 5 and $10 \% \mathrm{AE}$ and $75 \%$ at $20 \% \mathrm{AE}$. In contrast, when growing at $30 \% \mathrm{AE}$ almost half of the available sugars remain in the medium even after 7 days in culture. As was shown below (Table 1), in AW, 95\% of available sugars are used.

Although in the metabolic test we observed that $R h$. mucilaginosa can grow using all the sugars present as the sole $\mathrm{C}$ source (Additional file 1: Fig. S1b), in the AE this was not the case (Table 1). The monosaccharides glucose and fructose were efficiently consumed at all AE concentrations tested. Besides, the utilization of the tetrasaccharides stachyose was incomplete and only observed at 10 and $20 \% \mathrm{AE}$, and sucrose and mannitol content were not significantly used in at any AE concentration tested. As the concentration of $\mathrm{AE}$ increases, $R h$. mucilaginosa became more selective, at $30 \% \mathrm{AE}$ only glucose and fructose are consumed, and a large amount of sugars remain in the medium. At 30\% AE, the high levels of polyphenols present could act as inhibitors of some enzymes involved in the metabolization of disaccharides and oligosaccharides, thus precluding their utilization. Many natural polyphenols like flavonoids and phenolic alcohols can act as enzymes inhibitors with IC50 starting from $0.6 \mathrm{mg} / \mathrm{L}$ (Rasouli et al. 2017; Renda et al. 2018; Bhatia et al. 2019; Lim et al. 2019). Most of them are present in alperujo and since they are water soluble, they are expected to be present in the AE (Araújo et al. 2015; Rubio-Senent et al. 2013). Moreover, phenolic extracts obtained from olive oil were shown to inhibit $\alpha$-glucosidase activity in vitro with an IC50 between 60 and $350 \mathrm{mg} / \mathrm{L}$ (FigueiredoGonzález et al. 2019). Also, hydroxytyrosol a main phenolic alcohol present in alperujo, was shown to inhibit intestinal $\beta$-galactosidase and $\alpha$-glucosidase in diabetic rats (Hamden et al. 2010). In the present work, the polyphenol content is ranging from $500 \mathrm{mg} / \mathrm{L}$ to $3000 \mathrm{mg} / \mathrm{L}$, values much higher than those commonly reported to inhibit enzymes involved in the metabolization of more complex sugars; this fact could explain the lack of consumption of di- and oligo-saccharides observed. Interestingly, when $R h$. mucilaginosa grew on AW, it was able to metabolize all the sugars present in the medium and with high efficiency, despite the high polyphenol concentration.

Phytotoxicity of AW and AE against the germination of Lactuca sativa was studied next. As shown in Fig. 4a (empty bars), the number of germinated seeds depends on the $\mathrm{AE}$ concentration. Whereas at 5 and $10 \% \mathrm{AE}$, no deleterious effect is seen in the capacity of seeds to germinate, at 20,30\% AE and AW, the number of germinated seeds decreases up to $40 \%$. However, Rh. mucilaginosa growth does not impact the phytotoxicity of the $\mathrm{AE}$ at any AE concentration tested nor at AW (Fig. 4a, dotted bars). This is probably related to the fact that polyphenol content does not change either (Fig. 4b). Although polyphenols present in alperujo are reported to be phytotoxic, they are valuable compounds for diverse industries because of their antioxidant, antimicrobial and antitumoral properties (Araújo et al. 2015). Nowadays, a lot of research has been done to recover these compounds from olive wastes (Fernández-Prior et al. 2020; Fernández-Bolaños et al. 2002; Rubio-Senent et al. 2013; Serrano et al. 2017). Since polyphenol content does not change, it is still possible to recover these valuable compounds 

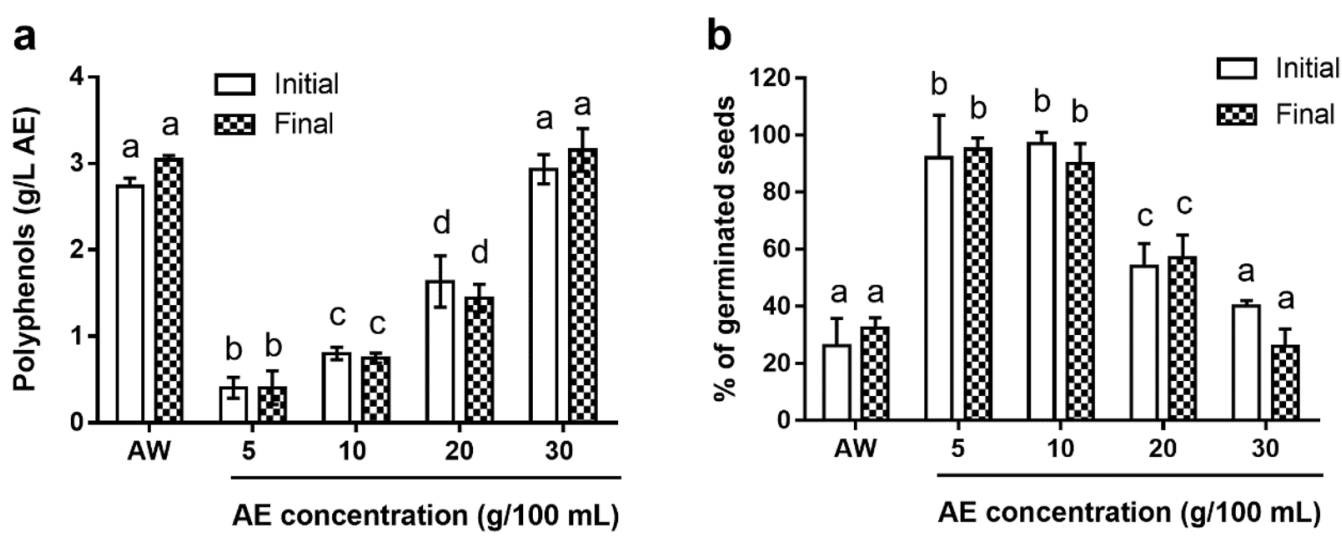

Fig. 4 Phytotoxicity of different mediums before and after Rh. mucilaginosa growth. a Polyphenol content of different AE before and after 4 days of Rh. mucilaginosa growth. $\mathbf{b}$ Percent of germinated seeds with different AE concentrations before and after 6 days of Rh. mucilaginosa growth. Bars show the mean and S.D. $(n=3)$; different letters indicate statistically significant differences $\left(p^{<} 0.01\right)$

even after $R h$. mucilaginosa growth and, thus, include the production of carotenoids compounds proposed here as a step in a revalorization chain for olive wastes.

\section{Carotenoid production by Rh. mucilaginosa}

Total carotenoid volumetric production significantly increases with $\mathrm{AE}$ concentration reaching a maximum at $20 \%$ and $30 \% \mathrm{AE}(5.5 \pm 1.0 \mathrm{mg} / \mathrm{L}$ and $4.9 \pm 0.5 \mathrm{mg} / \mathrm{L}$, respectively). However, when $R h$. mucilaginosa grows at AW, $7.3 \pm 0.6 \mathrm{mg} / \mathrm{L}$ of total carotenoids are obtained (Fig. 5a). At 10\% AE, the carotenoid increase could be related to faster yeast growth that renders more cells; however, at AW, 20 and 30\% AE, the increase in carotenoid content is probably reflecting the accumulation of carotenoids inside the cells. Indeed, comparing with initial inoculum, specific carotenoid content also increases at all AE concentrations and in AW (Fig. 5b, dot line) being higher during $R h$. mucilaginosa growth at AW or $30 \%$ AE. These results demonstrate that these mediums could be used as an induction medium for carotenoid production. The values obtained here are larger than previous reports of the same species isolated from soil, grown on synthetic media like Luria-Bertani, potatodextrose or yeast extract-peptone-dextrose $(189.2 \mu \mathrm{g} / \mathrm{g})$ (Yoo et al. 2016). However, they are similar to the carotenoids-specific production obtained by optimization of $R h$. mucilaginosa growth in diverse agro-industrial wastes (Sharma and Ghoshal 2020). At AW and 30\% AE, although Rh. mucilaginosa grew slower and carotenoid production is stimulated when compared with the other AE concentrations.

In addition to the increase in total carotenoid content, carotenoid profile changes depending on the medium too (Fig. $5 \mathrm{c}$ ). $\beta$-carotene increment does not vary among the mediums, except for AW. This indicates that other carotenoids are synthesized and are responsible for the further total carotenoid increase. It is well known that besides $\beta$-carotene, yeasts belonging to Rhodotorula genera produce torulene and torularhodin at different proportions depending on culture conditions (Tang et al. 2019; Moliné et al. 2012). These carotenoids can be tentatively identified by their characteristic UV/Vis spectrum and their retention time in RP-HPLC (Weber et al. 2007). Therefore, torularhodin, which has a carboxylic group and 14 conjugated double bonds (c.d.b.), is expected to have a shorter retention time and to absorb at a longer wavelength (Moliné et al. 2012) than torulene and $\beta$-carotene. Besides, the presence of the carboxylic group reduces the fine structure of the spectrum, which can be estimated by the ratio \%III/ II. Indeed, as shown in Additional file 1: Fig. S3, the first peak appearing (Retention time $=13.4 \mathrm{~min}$ ) bears the same UV/Vis spectrum as that reported for torularhodin, with a maximum at $498 \mathrm{~nm}$ and a poor fine structure $\left(\%_{\text {III/II }}=9\right)$. The second peak (Retention time $\left.=18.4 \mathrm{~min}\right)$ shows a spectrum with more defined bands of absorption $\left(\%_{\mathrm{II} / \mathrm{II}}=40\right)$ and a maximum at $490 \mathrm{~nm}$, as it is expected for torulene which has 13 c.d.b. and is not as polar as torularhodin. Lastly, peak 5 corresponds to $\beta$-carotene which is the less polar carotene and elutes at $20.6 \mathrm{~min}$ as the standard did (see Additional file 1: Figs. S3 and S4). Therefore, the main carotenoids produced are torulene, torularhodin and $\beta$-carotene. The sum of these represents about $70 \%$ of the total carotenoid content. Also, according to the culture condition, we found two minor peaks between torulene and $\beta$-carotene that most probably correspond to neurosporene and $\gamma$-carotene, according to its $\mathrm{UV} / \mathrm{Vis}$ spectrum, retention time and literature data of carotenoid composition of Rhodotorula yeasts (Takaichi and Maoka 2015; Weber et al. 2007; Davoli et al. 2004) and Additional file 1: Fig. S3). When growing in AW, torulene 

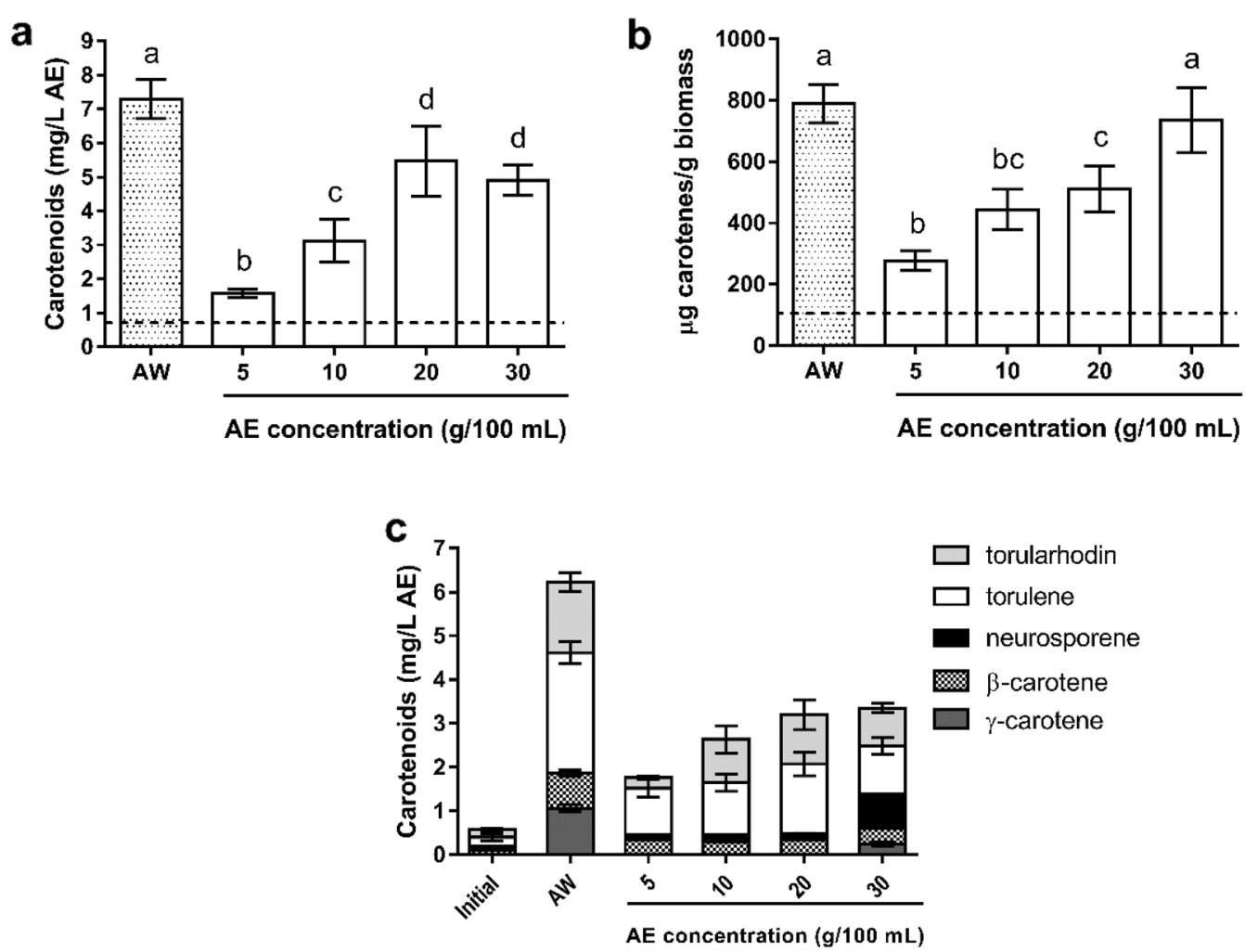

Fig. 5 Carotenoid production of Rh. mucilaginosa at different mediums. a Total carotenoid volumetric production; b specific carotenoid production. c Torularhodin, torulene, $\beta$-carotene and neurosporene contents obtained at mediums after 4 days of culture. Bars show the mean and S.D. $(n=3)$, different letters indicate statistically significant differences $\left(p^{<} 0.01\right)$

is the main carotenoid produced, followed by torularhodin and $\gamma$-carotene. No significant levels of neurosporene are detected probably indicating that the carotenoid biosynthetic route is very active. Among different AE concentrations, changes in carotenoid profiles are evident too. At $5 \%$ $\mathrm{AE}$, torulene represents $80 \%$ of the total carotenoid content. Its concentration reaches a maximum at $20 \% \mathrm{AE}$ and decreases again when Rh. mucilaginosa grows at 30\% AE. Torularhodin concentration, in contrast, became significantly higher than the initial inoculum at $10 \% \mathrm{AE}$. At $30 \%$ $\mathrm{AE}$, an accumulation of neurosporene is observed, probably indicating a slowdown in the carotenoid biosynthetic route (Fig. 5c). Neurosporene is located before the branching point in carotenoid biosynthesis, being a precursor of both torulene and $\beta$-carotene (Kot et al. 2018). The lack of change in $\beta$-carotene together with the increase in torulene observed indicates that $\mathrm{AE}$ favors the branch that goes to the synthesis of torulene and torularhodin. In AW, in contrast, both branches are stimulated. Carotenoids are involved in stress responses of microorganisms (Marova et al. 2004, 2010). Indeed, several works reported the enhanced production of torularhodin and torulene in response to oxidative stress, light exposure, shear stress, some chemicals and high salt concentrations ( $\mathrm{Li}$ et al. 2017; Sakaki et al. 2001; Davoli et al. 2004; Varmira et al. 2016). Alperujo is known to possess a high salt concentration and many toxic compounds like polyphenols, in addition to an unbalance nutrient composition, all factors stimulating carotenoid biosynthesis (Morillo et al. 2009).

\section{Conclusion}

The use of the liquid fraction of alperujo (AW) or an $\mathrm{AE}$ as a culture medium for carotenoid production by Rh. mucilaginosa was studied. Our results demonstrate that Rh. mucilaginosa can grow and produce carotenoids in both mediums without affecting the possibility of extracting other valuable compounds like polyphenols. According to the medium used, it may be possible to obtain a mix of carotenoids enriched in torulene, torularhodin and/or neurosporene. Thus, AW and AE seem to be promising, cheap and simple-toobtain mediums for producing carotenoids with industrial applications. 


\section{Supplementary information}

Supplementary information accompanies this paper at https://doi. org/10.1186/s40643-020-00341-7.

Additional file 1: Fig. S1. Sugar and nitrogen assimilation tests. (a) Rh. mucilaginosa was grown in base media with the specified compound as sole $\mathrm{N}$ source. Bars shown the mean and S.D. $(n=3)$ of optical density after 2 days in the culture at $30^{\circ} \mathrm{C}$ normalized against the O.D. of a culture grown in peptone. (b) Rh. mucilaginosa was grown in base media with the specified sugars as sole $C$ source. Bars shown the mean and S.D. $(n=3)$ of optical density after 2 days in the culture at $30^{\circ} \mathrm{C}$ normalized against the O.D. of a culture grown in glucose. ${ }^{*}$ denotes statistically significant differences with $p^{<} 0.01$. Fig. S2. HPLC separation of sugars. Chromatogram of sugars present in a sample of AE at $20 \%$ before (upper panel) and after (lower panel) 4 days of Rh. mucilaginosa growth. Fig. S3. HPLC separation of carotenoids. a) HPLC profile at $454 \mathrm{~nm}$ of total carotenoid extract from Rh. mucilaginosa at the beginning (dotted lines) or after 4 days of growing in AE 30\% (filled line). b) Absorption spectra of each peak obtained from DAD. Fig. S4. HPLC profile of $\beta$-carotene standard.

\section{Abbreviations}

TPOMW: Two-phase olive mill waste; AE: Aqueos extract of alperujo; AW: Alperujo water; BSA: Bovine serum albumin.

\section{Acknowledgements}

Not applicable.

\section{Authors' contributions}

CG performed the experiments. Analysis of the data. PSN Revised manuscript preparation. AC substantively revised the manuscript. VB designed the experiments. Interpretation of the results. Manuscript preparation. All authors read and approved the final manuscript.

\section{Funding}

This work was supported by "Agencia Nacional de Promoción Científica y Tecnológica" (ANPCyT, PICT 520-2016), "Consejo Nacional de Investigaciones Científicas y Técnicas" (CONICET, PIP 243-2013) and "Universidad Nacional del Sur" (UNS, PGI 24M/152, 2017), Argentina.

\section{Availability of data and materials}

The datasets used and/or analyzed during the current study are available from the corresponding author on reasonable request.

\section{Ethics approval and consent to participate}

Not applicable.

\section{Consent for publication}

Not applicable.

\section{Competing interests}

The authors declare that they have no competing interests.

\author{
Author details \\ 1 Planta Piloto de Ingeniería Química-PLAPIQUI (UNS-CONICET), Bahía Blanca, \\ Argentina. ${ }^{2}$ Departamento de Ingeniería Química, Universidad Nacional del \\ Sur (UNS), Bahía Blanca, Argentina. ${ }^{3}$ Instituto de Tecnología en Polímeros y \\ Nanotecnología (ITPN-UBA-CONICET), Facultad de Arquitectura, Diseño y \\ Urbanismo (FADU), Universidad de Buenos Aires (UBA), Ciudad Universitaria, \\ 1428 Buenos Aires, Argentina.
}

Received: 27 February 2020 Accepted: 23 September 2020 Published online: 30 September 2020

\section{References}

Araújo M, Pimentel FB, Alves RC, Oliveira MBP (2015) Phenolic compounds from olive mill wastes: health effects, analytical approach and application as food antioxidants. Trends Food Sci Technol 45:200-211

Bhatia A, Singh B, Arora R, Arora S (2019) In vitro evaluation of the a-glucosidase inhibitory potential of methanolic extracts of traditionally used antidiabetic plants. BMC Complement Altern Med 19:74

Borroni V, González MT, Carelli AA (2017) Bioproduction of carotenoid compounds using two-phase olive mill waste as the substrate. Process Biochem 54:128-134

Britton G, Young A (1993) Methods for the isolation and analysis of carotenoids. Carotenoids in photosynthesis. Springer, Berlin, pp 409-457

Colet R, Di Luccio M, Valduga E (2015) Fed-batch production of carotenoids by Sporidiobolus salmonicolor (CBS 2636): kinetic and stoichiometric parameters. Eur Food Res Technol 240:173-182

Davoli P, Mierau V, Weber RWS (2004) Carotenoids and fatty acids in red yeasts Sporobolomyces roseus and Rhodotorula glutinis. Appl Biochem Microbiol 40:392-397. https://doi.org/10.1023/B:ABIM.0000033917.57177.f2

Dermeche S, Nadour M, Larroche C et al (2013) Olive mill wastes: biochemical characterizations and valorization strategies. Process Biochem 48:1532-1552

Di Salvatore M, Carafa AM, Carratù G (2008) Assessment of heavy metals phytotoxicity using seed germination and root elongation tests: a comparison of two growth substrates. Chemosphere 73:1461-1464. https:// doi.org/10.1016/j.chemosphere.2008.07.061

Elfeky N, Elmahmoudy M, Zhang Y et al (2019) Lipid and carotenoid production by Rhodotorula glutinis with a combined cultivation mode of nitrogen, sulfur, and aluminium stress. Appl Sci 9:2444. https://doi. org/10.3390/app9122444

Elisashvili V, Kachlishvili E, Asatiani MD (2018) Efficient production of ligninmodifying enzymes and phenolics removal in submerged fermentation of olive mill by-products by white-rot basidiomycetes. Int Biodeterior Biodegrad 134:39-47. https://doi.org/10.1016/j.ibiod.2018.08.003

Fernández-Bolaños J, Rodríguez G, Rodríguez R et al (2002) Production in large quantities of highly purified hydroxytyrosol from liquid-solid waste of two-phase olive oil processing or "alperujo". J Agric Food Chem 50:6804-6811. https://doi.org/10.1021/jf011712r

Fernandez-Orozco R, Gallardo-Guerrero L, Hornero-Mendez D (2013) Carotenoid profiling in tubers of different potato (Solanum sp) cultivars: accumulation of carotenoids mediated by xanthophyll esterification. Food Chem 141:2864-2872. https://doi.org/10.1016/j.foodchem.2013.05.016

Fernández-Prior MÁ, Charfi A, Bermúdez-Oria A et al (2020) Deep eutectic solvents improve the biorefinery of alperujo by extraction of bioactive molecules in combination with industrial thermal treatments. Food Bioprod Process 121:131-142

Figueiredo-González M, Reboredo-Rodríguez P, González-Barreiro C et al (2019) The involvement of phenolic-rich extracts from Galician autochthonous extra-virgin olive oils against the a-glucosidase and a-amylase inhibition. Food Res Int 116:447-454

Giannoutsou E, Meintanis C, Karagouni A (2004) Identification of yeast strains isolated from a two-phase decanter system olive oil waste and investigation of their ability for its fermentation. Bioresour Technol 93:301-306

Gutfinger T (1981) Polyphenols in olive oils. J Am Oil Chem Soc 58:966-968

Hadjikinova R, Petkova N, Hadjikinov D et al (2017) Development and validation of HPLC-RID method for determination of sugars and polyols. J Pharm Sci 9:8

Hamden K, Allouche N, Jouadi B et al (2010) Inhibitory action of purified hydroxytyrosol from stored olive mill waste on intestinal disaccharidases and lipase activities and pancreatic toxicity in diabetic rats. Food Sci Biotechnol 19:439-447. https://doi.org/10.1007/s10068-010-0062-6

Jarboui R, Baati H, Fetoui F et al (2012) Yeast performance in wastewater treatment: case study of Rhodotorula mucilaginosa. Environ Technol 33:951-960. https://doi.org/10.1080/09593330.2011.603753 
Kaur P, Ghoshal G, Jain A (2019) Bio-utilization of fruits and vegetables waste to produce $\beta$-carotene in solid-state fermentation: characterization and antioxidant activity. Process Biochem 76:155-164

Killi D, Anlauf R, Kavdir Y, Haworth M (2014) Assessing the impact of agroindustrial olive wastes in soil water retention: implications for remediation of degraded soils and water availability for plant growth. Int Biodeterior Biodegrad 94:48-56. https://doi.org/10.1016/j.ibiod.2014.06.019

Kot AM, Błażejak S, Kurcz A et al (2017) Effect of initial pH of medium with potato wastewater and glycerol on protein, lipid and carotenoid biosynthesis by Rhodotorula glutinis. Electron J Biotechnol 27:25-31

Kot AM, Błażejak S, Gientka I et al (2018) Torulene and torularhodin: "new" fungal carotenoids for industry? Microb Cell Factories 17:49. https://doi. org/10.1186/s12934-018-0893-z

Leyton A, Flores L, Mäki-Arvela P et al (2019) Macrocystis pyrifera source of nutrients for the production of carotenoids by a marine yeast Rhodotorula mucilaginosa. J Appl Microbiol 127:1069-1079. https://doi. org/10.1111/jam.14362

Li C, Zhang N, Li B et al (2017) Increased torulene accumulation in red yeast Sporidiobolus pararoseus NGR as stress response to high salt conditions. Food Chem 237:1041-1047. https://doi.org/10.1016/j.foodc hem.2017.06.033

Lim J, Zhang X, Ferruzzi MG, Hamaker BR (2019) Starch digested product analysis by HPAEC reveals structural specificity of flavonoids in the inhibition of mammalian a-amylase and a-glucosidases. Food Chem 288:413-421

Lowry OH, Rosebrough NJ, Farr AL, Randall RJ (1951) Protein measurement with the Folin phenol reagent. J Biol Chem 193:265-275

Marova I, Breierova E, Koci R et al (2004) Influence of exogenous stress factors on production of carotenoids by some strains of carotenogenic yeasts. Ann Microbiol 54:73-86

Marova I, Carnecka M, Halienova A et al (2010) Production of carotenoid-/ ergosterol-supplemented biomass by red Yeast Rhodotorula glutinis grown under external stress. Food Technol Biotechnol 48:56-61

McWilliams A (2018) Global Carotenoids Market Research Report to 2022. In: bccresearch. https://www.bccresearch.com/market-research/food-andbeverage/the-global-market-for-carotenoids.html. Accessed 10 Sep 2019

Moliné M, Libkind D, van Broock M (2012) Production of torularhodin, torulene, and $\beta$-carotene by Rhodotorula yeasts. In: Barredo J-L (ed) Microbial carotenoids from fungi: methods and protocols. Humana Press, Totowa, pp 275-283

Monetta P, Bueno L, Cornejo V et al (2012) Short-term dynamics of soil chemical parameters after application of alperujo in high-density drip-irrigated olive groves in Argentina. Int J Environ Stud 69:578-588

Morillo JA, Aguilera M, Ramos-Cormenzana A, Monteoliva-Sánchez M (2006) Production of a metal-binding Exopolysaccharide by Paenibacillus jamilae using two-phase olive-mill waste as fermentation substrate. Curr Microbiol 53:189-193. https://doi.org/10.1007/s00284-005-0438-7

Morillo JA, Antizar-Ladislao B, Monteoliva-Sanchez M et al (2009) Bioremediation and biovalorisation of olive-mill wastes. Appl Microbiol Biotechnol 82:25-39. https://doi.org/10.1007/s00253-008-1801-y

Moya Ramírez I, Altmajer Vaz D, Banat IM et al (2016) Hydrolysis of olive mill waste to enhance rhamnolipids and surfactin production. Bioresour Technol 205:1-6. https://doi.org/10.1016/j.biortech.2016.01.016

Rasouli H, Hosseini-Ghazvini SM-B, Adibi H, Khodarahmi R (2017) Differential a-amylase/a-glucosidase inhibitory activities of plant-derived phenolic compounds: a virtual screening perspective for the treatment of obesity and diabetes. Food Funct 8:1942-1954

Renda G, Sari S, Barut B et al (2018) a-Glucosidase inhibitory effects of polyphenols from Geranium asphodeloides: inhibition kinetics and mechanistic insights through in vitro and in silico studies. Bioorganic Chem 81:545-552

Rodriguez-Amaya DB, Kimura M (2004) HarvestPlus handbook for carotenoid analysis. International Food Policy Research Institute, Washington

Rodriguez-Concepcion M, Avalos J, Bonet ML et al (2018) A global perspective on carotenoids: metabolism, biotechnology, and benefits for nutrition and health. Prog Lipid Res 70:62-93. https://doi.org/10.1016/j.plipr es.2018.04.004

Rubio-Senent F, Rodríguez-Gutiérrez G, Lama-Muñoz A, Fernández-Bolaños J (2013) Phenolic extract obtained from steam-treated olive oil waste: characterization and antioxidant activity. LWT-Food Sci Technol 54:114-124
Sakaki H, Nakanishi T, Tada A et al (2001) Activation of torularhodin production by Rhodotorula glutinis using weak white light irradiation. J Biosci Bioeng 92:294-297. https://doi.org/10.1016/S1389-1723(01)80265-6

Schneider T, Graeff-Hönninger S, French WT et al (2013) Lipid and carotenoid production by oleaginous red yeast Rhodotorula glutinis cultivated on brewery effluents. Energy 61:34-43. https://doi.org/10.1016/j.energ y.2012.12.026

Serrano A, Fermoso FG, Alonso-Fariñas B et al (2017) Olive mill solid waste biorefinery: high-temperature thermal pre-treatment for phenol recovery and biomethanization. J Clean Prod 148:314-323. https://doi. org/10.1016/j.jclepro.2017.01.152

Sharma R, Ghoshal G (2020) Optimization of carotenoids production by Rhodotorula mucilaginosa (MTCC-1403) using agro-industrial waste in bioreactor: a statistical approach. Biotechnol Rep 25:e00407

Takaichi S, Maoka T (2015) Identification and spectroscopic characterization of neurosporene. Biotechnol Lett 37:2027-2031. https://doi.org/10.1007/ s10529-015-1884-3

Tang W, Wang Y, Zhang J et al (2019) Biosynthetic pathway of carotenoids in Rhodotorula and strategies for enhanced their production. J Microbiol Biotechnol 29:507-517

Unrean P (2016) Bioprocess modelling for the design and optimization of lignocellulosic biomass fermentation. Bioresour Bioprocess 3:1. https:// doi.org/10.1186/s40643-015-0079-z

Urnau L, Colet R, Reato PT et al (2019) Use of low-cost agro-industrial substrate to obtain carotenoids from Phaffia rhodozyma in a Bioreactor. Ind Biotechnol 15:25-34. https://doi.org/10.1089/ind.2018.0027

Varmira K, Habibi A, Bahramian E, Jamshidpour S (2016) Progressive agents for improvement of carotenogenesis in Rhodotorula rubra. J Adv Food Sci Technol 3:70-78

Weber RWS, Anke H, Davoli P (2007) Simple method for the extraction and reversed-phase high-performance liquid chromatographic analysis of carotenoid pigments from red yeasts (Basidiomycota, Fungi). J Chromatogr A 1145:118-122. https://doi.org/10.1016/j.chroma.2007.01.052

Wickerham LJ (1946) A Critical Evaluation of the Nitrogen Assimilation Tests Commonly Used in the Classification of Yeasts. J Bacteriol 52:293-301

Wickerham LJ, Burton KA (1948) Carbon assimilation tests for the classification of yeasts. J Bacteriol 56:363

Yoo AY, Alnaeeli M, Park JK (2016) Production control and characterization of antibacterial carotenoids from the yeast Rhodotorula mucilaginosa AY-01. Process Biochem 51:463-473

Zerva A, Papaspyridi L-M, Christakopoulos P, Topakas E (2017) Valorization of olive mill wastewater for the production of $\beta$-glucans from selected basidiomycetes. Waste Biomass Valorization 8:1721-1731. https://doi. org/10.1007/s12649-017-9839-7

Zoz L, Carvalho JC, Soccol VT et al (2015) Torularhodin and torulene: bioproduction, properties and prospective applications in food and cosmetics-a review. Braz Arch Biol Technol 58:278-288

\section{Publisher's Note}

Springer Nature remains neutral with regard to jurisdictional claims in published maps and institutional affiliations.

\section{Submit your manuscript to a SpringerOpen ${ }^{\circ}$ journal and benefit from:}

- Convenient online submission

- Rigorous peer review

- Open access: articles freely available online

- High visibility within the field

- Retaining the copyright to your article

Submit your next manuscript at springeropen.com 